\title{
Customization in an Endogenous-Timing Game with Vertical Differentiation
}

\author{
Oksana Loginova* X. Henry Wang ${ }^{\dagger}$
}

September 22, 2008

\begin{abstract}
We study customization in a duopoly game in which the firms' products have different qualities. Whether customization choices are made simultaneously or sequentially is endogenously determined. Specifically, the customization stage of the game involves two periods. Each firm either selects its product type in period 1 or postpones this decision to period 2 . We show that both quality and endogenous timing play important roles in determining the equilibrium outcome. Customization occurs only if the quality difference is sufficiently large. Endogenous timing sometimes enables the firms to achieve an outcome that is Pareto superior to that if they were to make their customization choices simultaneously. Although the higher quality firm is more likely to customize, endogenous timing sometimes enables the lower quality firm to obtain an advantage that it would not have under simultaneous customization choices.
\end{abstract}

JEL classification: D43, L13, C72

Keywords: customization, horizontal differentiation, vertical differentiation, endogenous timing

\footnotetext{
${ }^{*}$ Department of Economics, University of Missouri-Columbia, 333 Professional Building, Columbia, MO 65211, U.S.A., e-mail: loginovao@missouri.edu (corresponding author)

${ }^{\dagger}$ Department of Economics, University of Missouri-Columbia, 318 Professional Building, Columbia, MO 65211, U.S.A., e-mail: wangx@missouri.edu
} 


\section{Introduction}

Customization is a flexible computer-aided technology designed to produce individually tailored products without significantly compromising cost efficiency. For example, Dell builds to order notebook and desktop computers; NikeID.com allows consumers to create their most preferred athletic pair of shoes; Timbuk2 customizes messenger bags and backpacks; apparel vendor LandsEnd.com offers custom-crafted pants and shirts.

Most of the existing theoretical literature on customization adopt one-dimensional horizontal differentiation settings (e.g., Dewan et al. 2003, Syam and Kumar 2006, Alexandrov 2008, and Mendelson and Parlaktürk 2008). Customization enables consumers to get their ideal products represented by their locations in the product attribute space. Firms are symmetrical and make symmetric choices in equilibrium. ${ }^{1}$ Even though many important aspects of customization are captured by these studies, important issues have yet to be examined. Casual empiricism indicates that (i) some firms customize, some do not; (ii) firms may not make their customization choices at the same time; and (iii) higher quality firms are more likely to offer customization. The goal of the present paper as well as Loginova and Wang (2008) is to incorporate these observations into product customization competition.

We consider an industry in which products are characterized by variety and quality. Variety is a horizontal attribute and quality is a vertical attribute. ${ }^{2}$ Consumer preferences are heterogenous in two dimensions. In particular, each consumer has a most preferred variety and a quality valuation. There are two firms that initially produce standard products located at the end points of the variety space. The firms are asymmetrical due to having different qualities. Customization provides ideal varieties for consumers but has no effect on product qualities. The model has two stages, the customization stage followed by the pricing stage. In Loginova and Wang (2008), the customization stage involves a single period in which the firms simultaneously decide whether to customize their products. In the present paper, the customization stage unfolds in two periods. Each firm either selects its product type in period 1 or postpones this decision to period 2. Thus, whether the customization choices are made simultaneously or sequentially is endogenously determined. While Loginova and Wang (2008) addresses issues related to observations (i) and (iii) above, this paper concerns all three observations by introducing endogenous timing into the customization stage.

Industrial organization economists have long been interested in whether firms choose their prices and/or quantities simultaneously or sequentially. Hamilton and Slutsky $(1990,1993)$ were the first to introduce a model in which the determination of simultaneity versus sequentiality of moves is endogenous. ${ }^{3}$ The game structure for the customization stage in this paper follows Hamilton and Slutsky (1993), in which the basic game is given by a $2 \times 2$ matrix and the extended game has two periods. This is the simplest possible setup to model firms' flexibility in choosing when to move.

\footnotetext{
${ }^{1}$ An exception is Mendelson and Parlaktürk (2008) in which asymmetric firms are considered.

${ }^{2}$ Our basic model is based on the literature that combines horizontal and vertical differentiation, e.g., Economides (1989) and Neven and Thisse (1990).

${ }^{3}$ Many subsequent studies have examined endogenous timing and related issues in standard price and/or quantity duopoly games, e.g., Matsumura (1999) and Amir and Stepanova (2006).
} 
The paper on customization that is closest to ours is Syam et al. (2005). In all three papers the consumer space is two-dimensional. Syam et al. (2005) endow products with two horizontal attributes, for which consumers have heterogeneous preferences. The firms are initially maximally differentiated with respect to both attributes. They first simultaneously choose whether to customize both, one, or none of the attributes, then compete in prices. The key difference between Syam et al. (2005) and our studies is that they work with ex ante symmetric firms and examine how the possibility of customizing multiple attributes affect customization choices, whereas we work with asymmetric firms and focus on the roles of quality difference and endogenous timing in customization competition.

Another closely related paper is Bernhardt et al. (2007), in which ex ante symmetric firms first acquire information about consumers and then customize their products as best as they can to match consumer needs. Similar to our papers, consumer preferences are two-dimensional, corresponding to two attributes of the product, and the second attribute - brand loyalty - cannot be customized. There are two main differences between Bernhardt et al. (2007) and our studies. First, Bernhardt et al. (2007) emphasize the cost side of customization, whereas our focus is shifted towards the strategic effects of customization. Second, brand loyalty is a horizontal attribute, not vertical as quality in our papers is.

Several other papers have studied customization using a one-dimensional consumer space. Dewan et al. (2003) assume that customizing firms price discriminate. Syam and Kumar (2006) examine the role of standard products in customization competition. Alexandrov (2008) extends Salop's (1979) model in which firms can offer interval-long adjustable "fat" products. While these three studies assume symmetric firms, in Mendelson and Parlaktürk (2008) one firm has margin advantage (higher difference between reservation price and unit cost) over the other.

Our papers as well as Syam et al. (2005) model customization as zero-one decisions, so that all customers of a customizing firm get their most preferred varieties. In contrast, all the other papers mentioned above treat customization as continuous choices. Both approaches match aspects of reality and have their advantages. With zero-one decisions, more attention can be devoted to the strategic effects of customization. With continuous customization choices, one can focus on how efficiency considerations determine the range of customization.

Our equilibrium analysis shows that quality difference and endogenous timing play important roles in determining the equilibrium outcome. In particular, no firm will customize if the quality difference is small, regardless of the fixed cost of customization. Intuitively, customization by one or both firms makes their products less differentiated, thus intensifying price competition. The smaller is the quality difference, the tougher is price competition. For sufficiently large quality differences, customization by one or both firms may occur. Because the high quality firm benefits more from customization than the low quality firm, the high quality firm is more likely to customize.

We show that the endogeneity of timing in the customization stage is an additional strategic tool for the firms. It sometimes enables the firms to achieve an outcome that is Pareto superior to that if they were to make their customization decisions simultaneously. While in Loginova and Wang (2008) the low quality firm never customizes alone, in the present model it can obtain an advantage 
by becoming the first and only firm to customize in some circumstances.

The rest of the paper is organized as follows. In the next section we introduce the model. In Section 3 the pricing stage of the game is analyzed. In Section 4 we study the firms' customization choices. Concluding remarks are provided in Section 5. Proofs of all lemmas and propositions are relegated to the Appendix.

\section{The Model}

Consider a market in which each product $i$ is characterized by its variety $x_{i} \in[0,1]$ and its quality $q_{i} \geq 0$. The first characteristic corresponds to horizontal differentiation and the second to vertical differentiation. Consumers are heterogenous in two dimensions. Each consumer has a most preferred variety $x \in[0,1]$ and a quality valuation $y \in[0,1]$. A consumer of type $(x, y)$ derives the following utility from buying one unit of product $i$ :

$$
v+q_{i} y-t\left|x-x_{i}\right|-p_{i}
$$

where $v$ is a positive constant, $t$ is a preference parameter, and $p_{i}$ is the price of product $i$. Consumers as represented by $(x, y)$ are uniformly distributed over the unit square $[0,1] \times[0,1]$ with a total mass of 1 . We assume that $v$ is large enough for all consumers to find a product that yields positive payoff in equilibrium.

There are two firms, $\mathrm{A}$ and $\mathrm{B}$, operating with zero marginal costs of production. Initially, firm A offers a single (standard) product of quality $q_{A}$ and variety $x_{A}=0$, whereas firm B offers a single product of quality $q_{B}>q_{A}$ and variety $x_{B}=1$. That is, firm $\mathrm{B}$ is the higher quality firm and the two firms have maximum variety differentiation.

We will normalize $t$ to 1 . This amounts to a monotonic transformation of preferences. The utilities of a consumer of type $(x, y)$ from buying firm A's and firm B's standard products are

$$
v+q_{A} y-x-p_{A}
$$

and

$$
v+q_{B} y-(1-x)-p_{B},
$$

respectively.

Investing $K \geq 0$ into product-customization technology allows a firm to produce a product that exactly matches a given consumer's preferred variety. The utilities of type $(x, y)$ from buying firm A's and firm B's customized products are

$$
v+q_{A} y-p_{A}
$$

and

$$
v+q_{B} y-p_{B}
$$




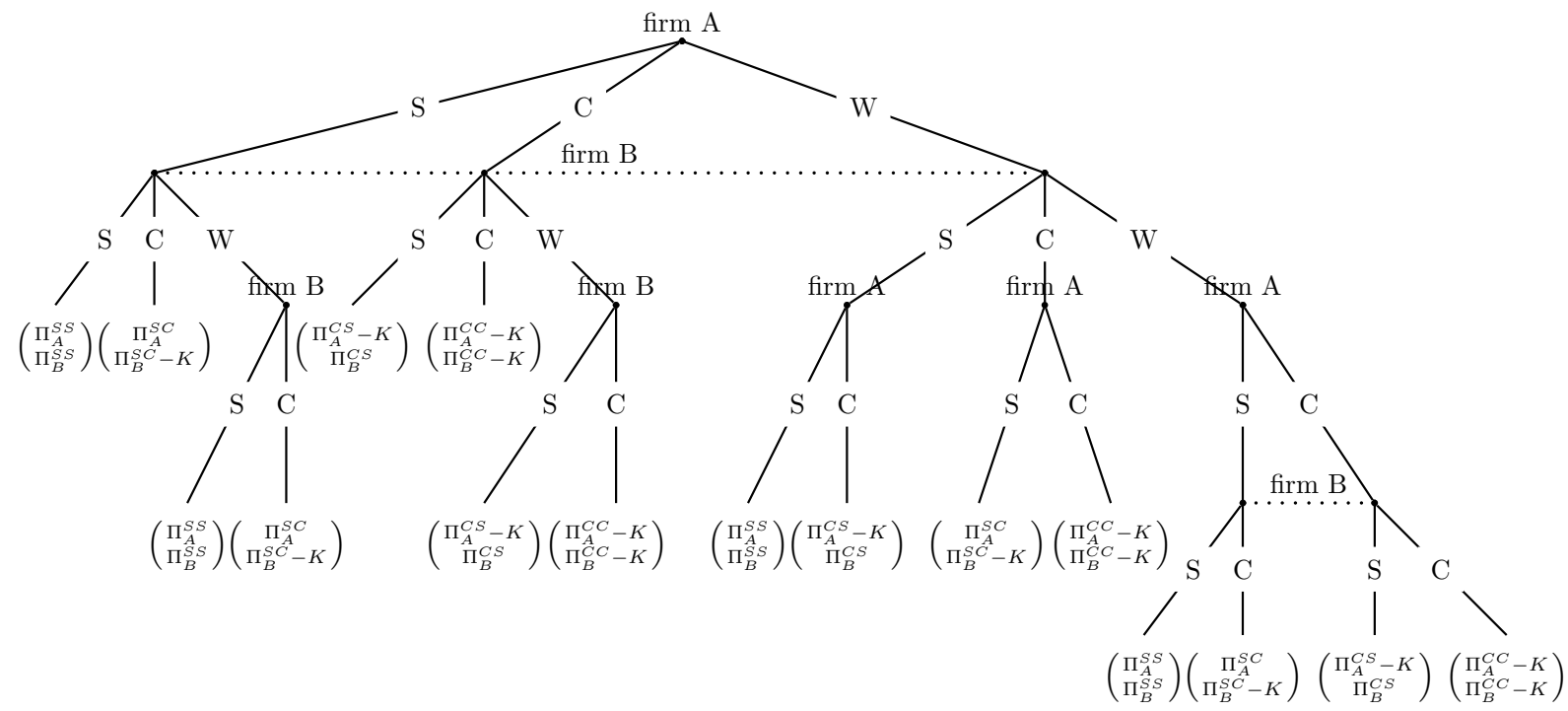

Figure 1: Customization stage

There are two stages in the game. The first stage - customization stage - unfolds in two periods. In period 1 the firms choose simultaneously between selecting their product types (standard or customized) this period or wait until period 2. We use S, C, and $\mathrm{W}$ to denote these three choices. If both firms selected their product types in period 1, the game proceeds to the second stage. If one firm chose to wait in period 1 , it must select its product type in period 2. If both firms chose to wait in period 1 , they simultaneously select their product types in period 2 . A firm selecting a customized product incurs fixed cost $K$.

The second stage of the game is the pricing stage. The firms choose simultaneously their prices, taking as given the product types selected in the customization stage. Consumers observe prices and make their purchasing decisions.

We adopt the subgame perfect Nash equilibrium and use backward induction to solve the game. Figure 1 depicts the reduced extensive form of the game. In this figure $\Pi_{A}$ and $\Pi_{B}$ are the firms' equilibrium profits in the pricing stage (presented in the next section). Superscripts SS, CS, SC, and CC correspond to the four possible outcomes of the first stage.

\section{Analysis of the Pricing Stage}

In this section we derive the firms' equilibrium prices and profits in the second stage of the game.

\subsection{Equilibrium Prices Following SS}

Suppose both firms selected standard products in the customization stage. Utilities from firm A's and firm B's standard products are given by (1) and (2). Therefore, for a given level of quality 


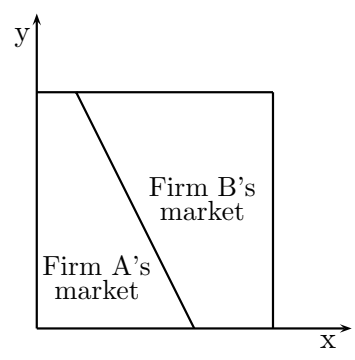

(a)

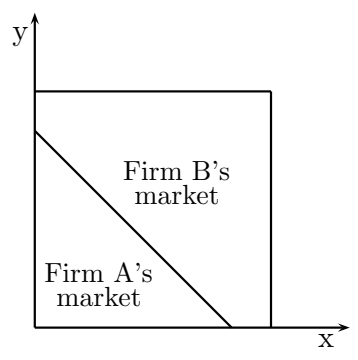

(b)

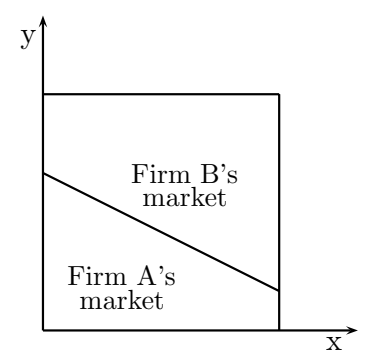

(c)

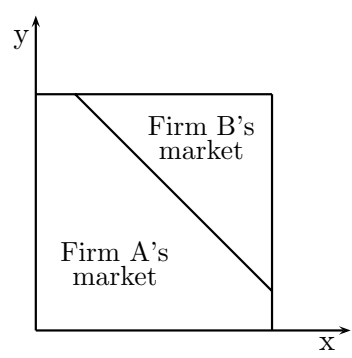

(d)

Figure 2: Indifference line and market areas

valuation $y$, the marginal consumer type in terms of variety $x$ is

$$
\widehat{x}(y)=\frac{1}{2}\left(1-q y+p_{B}-p_{A}\right),
$$

where

$$
q \equiv q_{B}-q_{A}
$$

denotes the quality difference between products of firm B and firm A. For any $y \in[0,1]$, consumers in the interval $x \in[0, \widehat{x}(y)]$ will purchase from firm A, whereas those with $x \in(\widehat{x}(y), 1]$ will purchase from firm B. There are four possible positions for the indifference line (5), as illustrated in Figure 2.

Let $D_{A}\left(p_{A}, p_{B}\right)$ and $D_{B}\left(p_{A}, p_{B}\right)$ denote the demand functions of firms $\mathrm{A}$ and $\mathrm{B}$. The expressions for these functions depend on the position of the indifference line. ${ }^{4}$ The firms choose simultaneously $p_{A}$ and $p_{B}$ to maximize their profits,

$$
\Pi_{A}\left(p_{A}, p_{B}\right)=D_{A}\left(p_{A}, p_{B}\right) p_{A}
$$

and

$$
\Pi_{B}\left(p_{A}, p_{B}\right)=D_{B}\left(p_{A}, p_{B}\right) p_{B}
$$

Lemma 1 (Equilibrium prices and profits following SS). Suppose both firms selected standard products in the first stage, then the equilibrium prices and profits in the second stage are as follows.

(i) If $q \leq 3 / 2$,

$$
\left\{\begin{array} { l } 
{ p _ { A } ^ { S S } = 1 - \frac { 1 } { 6 } q } \\
{ p _ { B } ^ { S S } = 1 + \frac { 1 } { 6 } q }
\end{array} \text { and } \left\{\begin{array}{l}
\Pi_{A}^{S S}=\frac{1}{2}\left(1-\frac{1}{6} q\right)^{2} \\
\Pi_{B}^{S S}=\frac{1}{2}\left(1+\frac{1}{6} q\right)^{2}
\end{array}\right.\right.
$$

(ii) If $q \in(3 / 2,3]$,

$$
\left\{\begin{array} { l } 
{ p _ { A } ^ { S S } = \frac { 1 } { 8 } ( 1 + \sqrt { 1 + 1 6 q } ) } \\
{ p _ { B } ^ { S S } = \frac { 1 } { 8 } ( - 5 + 3 \sqrt { 1 + 1 6 q } ) }
\end{array} \text { and } \left\{\begin{array}{l}
\Pi_{A}^{S S}=\frac{1}{q}\left(\frac{1+\sqrt{1+16 q}}{8}\right)^{3} \\
\Pi_{B}^{S S}=\left(1-\frac{1}{q}\left(\frac{1+\sqrt{1+16 q}}{8}\right)^{2}\right) \frac{-5+3 \sqrt{1+16 q}}{8}
\end{array}\right.\right.
$$

\footnotetext{
${ }^{4}$ The details are provided in the proof of Lemma 1.
} 
(iii) If $q>3$,

$$
\left\{\begin{array} { l } 
{ p _ { A } ^ { S S } = \frac { 1 } { 3 } q } \\
{ p _ { B } ^ { S S } = \frac { 2 } { 3 } q }
\end{array} \text { and } \left\{\begin{array}{l}
\Pi_{A}^{S S}=\frac{1}{9} q \\
\Pi_{B}^{S S}=\frac{4}{9} q
\end{array}\right.\right.
$$

It is easy to verify that the corresponding prices and profits vary continuously as $q$ changes. Case (i) corresponds to Figure 2(a), case (ii) to 2(b), and case (iii) to 2(c). Figure 2(d) does not arise in equilibrium. In all three cases the higher quality firm B sets a higher price, serves a larger market area, and earns a higher profit than firm A.

\subsection{Equilibrium Prices Following CS}

Suppose firm A selected a customized product and firm B selected a standard product in the customization stage. Utilities from firm A's customized product and firm B's standard product are given by (3) and (2). Therefore, for a given $y$, the marginal consumer type in terms of $x$ is

$$
\widehat{x}(y)=1-q y+p_{B}-p_{A},
$$

from which we can calculate the demand and profit functions for the firms. Lemma 2 presents the equilibrium following CS.

Lemma 2 (Equilibrium prices and profits following CS). Suppose firm A selected a customized product and firm $B$ selected a standard product in the first stage, then the equilibrium prices and profits in the second stage are as follows.

(i) If $q \leq 1$,

$$
\left\{\begin{array} { l } 
{ p _ { A } ^ { C S } = \frac { 2 } { 3 } - \frac { 1 } { 6 } q } \\
{ p _ { B } ^ { C S } = \frac { 1 } { 3 } + \frac { 1 } { 6 } q }
\end{array} \text { and } \left\{\begin{array}{l}
\Pi_{A}^{C S}=\left(\frac{2}{3}-\frac{1}{6} q\right)^{2} \\
\Pi_{B}^{C S}=\left(\frac{1}{3}+\frac{1}{6} q\right)^{2}
\end{array}\right.\right.
$$

(ii) If $q>1$,

$$
\left\{\begin{array} { l } 
{ p _ { A } ^ { C S } = \frac { 1 } { 3 } q + \frac { 1 } { 6 } } \\
{ p _ { B } ^ { C S } = \frac { 2 } { 3 } q - \frac { 1 } { 6 } }
\end{array} \text { and } \left\{\begin{array}{l}
\Pi_{A}^{C S}=\frac{1}{q}\left(\frac{1}{3} q+\frac{1}{6}\right)^{2} \\
\Pi_{B}^{C S}=\frac{1}{q}\left(\frac{2}{3} q-\frac{1}{6}\right)^{2}
\end{array}\right.\right.
$$

The pricing equilibrium following CS leads to only two positions of the indifference line. Case (i) corresponds to Figure 2(a). Customization enables firm A to overcome its quality disadvantage. Firm A's price, market size, and profit are higher than those of firm B. Case (ii) corresponds to Figure 2(c). When the quality difference is large, firm A's price, market size, and profit are lower than those of firm B.

\subsection{Equilibrium Prices Following SC}

Suppose firm A selected a standard product and firm B selected a customized product in the customization stage. Utilities from firm A's standard product and firm B's customized product are given by (1) and (4). Therefore, for a given $y$, the marginal consumer type in terms of $x$ is

$$
\widehat{x}(y)=-q y+p_{B}-p_{A} .
$$


The next lemma presents the equilibrium following SC.

Lemma 3 (Equilibrium prices and profits following SC). Suppose firm A selected a standard product and firm $B$ selected a customized product in the first stage, then the equilibrium prices and profits in the second stage are as follows.

(i) If $q \leq 1 / 2$,

$$
\left\{\begin{array} { l } 
{ p _ { A } ^ { S C } = \frac { 1 } { 3 } - \frac { 1 } { 6 } q } \\
{ p _ { B } ^ { S C } = \frac { 2 } { 3 } + \frac { 1 } { 6 } q }
\end{array} \text { and } \left\{\begin{array}{l}
\Pi_{A}^{S C}=\left(\frac{1}{3}-\frac{1}{6} q\right)^{2} \\
\Pi_{B}^{S C}=\left(\frac{2}{3}+\frac{1}{6} q\right)^{2}
\end{array}\right.\right.
$$

(ii) If $q \in(1 / 2,2]$,

$$
\left\{\begin{array} { l } 
{ p _ { A } ^ { S C } = \frac { 1 } { 4 } \sqrt { 2 q } } \\
{ p _ { B } ^ { S C } = \frac { 3 } { 4 } \sqrt { 2 q } }
\end{array} \text { and } \left\{\begin{array}{l}
\Pi_{A}^{S C}=\frac{1}{16} \sqrt{2 q} \\
\Pi_{B}^{S C}=\frac{9}{16} \sqrt{2 q}
\end{array}\right.\right.
$$

(iii) If $q>2$,

$$
\left\{\begin{array} { l } 
{ p _ { A } ^ { S C } = \frac { 1 } { 3 } q - \frac { 1 } { 6 } } \\
{ p _ { B } ^ { S C } = \frac { 2 } { 3 } q + \frac { 1 } { 6 } }
\end{array} \text { and } \left\{\begin{array}{l}
\Pi_{A}^{S C}=\frac{1}{q}\left(\frac{1}{3} q-\frac{1}{6}\right)^{2} \\
\Pi_{B}^{S C}=\frac{1}{q}\left(\frac{2}{3} q+\frac{1}{6}\right)^{2}
\end{array}\right.\right.
$$

Case (i) corresponds to Figure 2(a), case (ii) to 2(b), and case (iii) to 2(c). Firm B's quality advantage is reinforced by customization, pushing the critical values lower compared to those in Lemma 1.

\subsection{Equilibrium Prices Following CC}

Suppose both firms selected customized products in the first stage. Utilities from firm A's and firm B's customized products are given by (3) and (4). Therefore, consumers with $y \leq\left(p_{B}-p_{A}\right) / q$ will purchase from firm A, those with $y>\left(p_{B}-p_{A}\right) / q$ will purchase from firm $\mathrm{B}$. The firms' profit functions are

$$
\Pi_{A}\left(p_{A}, p_{B}\right)=\frac{1}{q}\left(p_{B}-p_{A}\right) p_{A} \quad \text { and } \quad \Pi_{B}\left(p_{A}, p_{B}\right)=\frac{1}{q}\left(q+p_{A}-p_{B}\right) p_{B}
$$

The profit maximizing first-order conditions

$$
\left\{\begin{array}{l}
p_{B}-2 p_{A}=0 \\
q+p_{A}-2 p_{B}=0
\end{array}\right.
$$

lead immediately to the following lemma.

Lemma 4 (Equilibrium prices and profits following CC). Suppose both firms selected customized products in the first stage, then the equilibrium prices and profits in the second stage are given by:

$$
\left\{\begin{array} { l } 
{ p _ { A } ^ { C C } = \frac { 1 } { 3 } q } \\
{ p _ { B } ^ { C C } = \frac { 2 } { 3 } q }
\end{array} \text { and } \left\{\begin{array}{l}
\Pi_{A}^{C C}=\frac{1}{9} q \\
\Pi_{B}^{C C}=\frac{4}{9} q
\end{array}\right.\right.
$$


In equilibrium the indifference line is horizontal at $1 / 3$ from the bottom side of the unit square. That is, firm A serves all consumers with quality valuations less than $1 / 3$, and firm $B$ serves the rest. The result here is the same as in the standard model of vertical differentiation.

\section{Analysis of the Customization Stage}

It is left to analyze the customization stage depicted in Figure 1, further referred to as the endogenoustiming game. It is crucial to first consider the simultaneous game in which product selection takes place in a single period (i.e., waiting is not an option).

\subsection{The Simultaneous Game}

The normal form of the simultaneous game is

\section{Firm B}

\begin{tabular}{cc|c|c}
\multirow{2}{*}{ Firm A } & $\mathrm{S}$ & $\mathrm{C}$ \\
\cline { 2 - 4 } & $\mathrm{S}$ & $\Pi_{A}^{S S}, \Pi_{B}^{S S}$ & $\Pi_{A}^{S C}, \Pi_{B}^{S C}-K$ \\
\cline { 2 - 4 } & $\mathrm{C}$ & $\Pi_{A}^{C S}-K, \Pi_{B}^{C S}$ & $\Pi_{A}^{C C}-K, \Pi_{B}^{C C}-K$
\end{tabular}

Note that all possible payoffs of the endogenous-timing game, shown in Figure 1, appear in this matrix. ${ }^{5}$ It follows that

$$
\left.\begin{array}{l}
(\mathrm{S}, \mathrm{S}) \\
(\mathrm{C}, \mathrm{S}) \\
(\mathrm{S}, \mathrm{C}) \\
(\mathrm{C}, \mathrm{C})
\end{array}\right\} \text { is a Nash equilibrium if }\left\{\begin{array}{l}
K \geq \max \left\{c_{1}, r_{1}\right\} \\
K \in\left[r_{2}, c_{1}\right] \\
K \in\left[c_{2}, r_{1}\right] \\
K \leq \min \left\{c_{2}, r_{2}\right\}
\end{array}\right.
$$

where

$$
c_{1} \equiv \Pi_{A}^{C S}-\Pi_{A}^{S S} \quad \text { and } \quad c_{2} \equiv \Pi_{A}^{C C}-\Pi_{A}^{S C}
$$

denote firm A's change in gross profit in the two columns, and

$$
r_{1} \equiv \Pi_{B}^{S C}-\Pi_{B}^{S S} \quad \text { and } \quad r_{2} \equiv \Pi_{B}^{C C}-\Pi_{B}^{C S}
$$

denote firm B's change in gross profit in the two rows.

The inequality

$$
\max \left\{c_{1}, c_{2}\right\}<\min \left\{r_{1}, r_{2}\right\}
$$

holds for any value of $q$. Detailed expressions for $c_{1}, c_{2}, r_{1}$, and $r_{2}$ as functions of $q$ and the proof of (9) are provided in the Appendix. Inequality (9) stipulates that the low quality firm A always gains less from customization than the high quality firm B. It follows that the simultaneous game

\footnotetext{
${ }^{5}$ Matrix (8) is also a normal form representation of the period 2 subgame following the choice of W by both firms in the endogenous-timing game.
} 


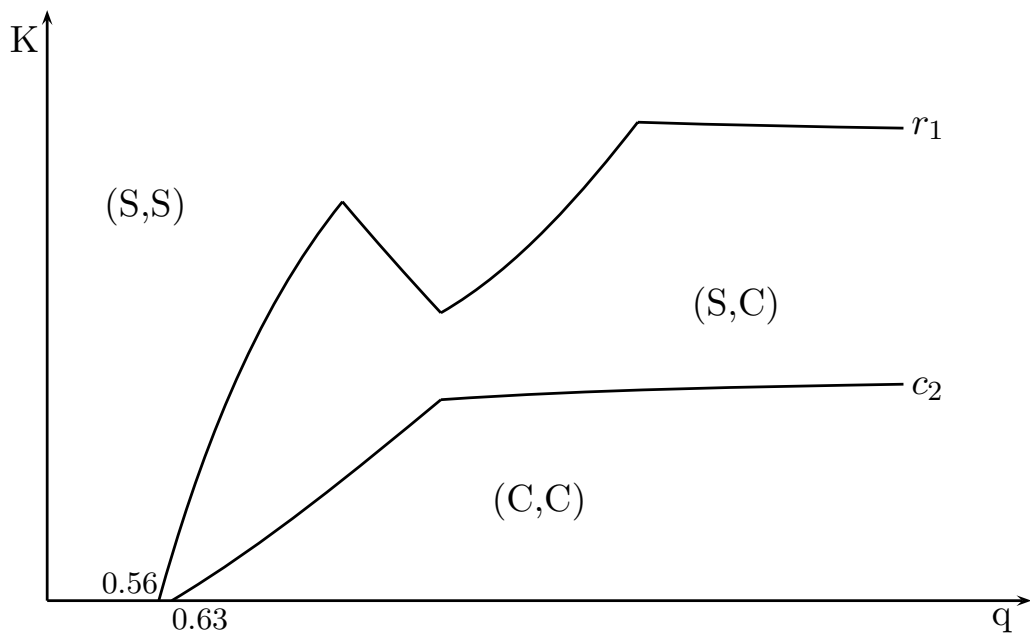

Figure 3: Customization choices in the simultaneous game

has a unique Nash equilibrium except for $K=c_{2}$ and $K=r_{1}$. For ease of presentation without affecting the results, we will select $(\mathrm{C}, \mathrm{C})$ as the Nash equilibrium at $K=c_{2}$ and $(\mathrm{S}, \mathrm{C})$ at $K=r_{1}$. Accordingly,

$$
\left.\begin{array}{l}
(\mathrm{S}, \mathrm{S}) \\
(\mathrm{S}, \mathrm{C}) \\
(\mathrm{C}, \mathrm{C})
\end{array}\right\} \text { is the Nash equilibrium if }\left\{\begin{array}{l}
K>r_{1} \\
K \in\left(c_{2}, r_{1}\right] \\
K \leq c_{2}
\end{array}\right.
$$

as shown in Figure 3. Stated in terms of $q$ and $K$, we have the following proposition.

Proposition 1 (Equilibrium customization choices in the simultaneous game). The following holds for the firms' equilibrium customization choices in the simultaneous game.

(i) If $q \leq 0.56$ then the Nash equilibrium is $(S, S)$ for any value of $K$.

(ii) If $q \in(0.56,0.63]$ then the Nash equilibrium is $(S, C)$ for $K \leq r_{1}$ and $(S, S)$ for $K>r_{1}$.

(iii) If $q>0.63$ then the Nash equilibrium is $(C, C)$ for $K \leq c_{2},(S, C)$ for $K \in\left(c_{2}, r_{1}\right]$, and $(S, S)$ for $K>r_{1}$.

Based on this proposition, customization can occur in the simultaneous game only if $q>0.56$. This is true even if customization is costless. For $q>0.56$, as customization becomes more expensive ( $K$ increases), the equilibrium customization choices change from both firms customizing to only firm B customizing, and to no firm customizing. Note that the low quality firm A never customizes alone.

The intuition behind the results of Proposition 1 is as follows. Customization by one or both firms makes their products less differentiated, intensifying price competition. The smaller is the quality difference, the tougher is price competition. Therefore, when $q$ is small, the firms do not customize in order to avoid a price war. When $q$ is large, one or both firms customize to take advantage of consumers' desires for ideal varieties. 
Before moving to the endogenous-timing game, we present the following two lemmas.

Lemma 5 (Outcome SS versus outcome CC). $\Pi_{A}^{S S} \geq \Pi_{A}^{C C}$ and $\Pi_{B}^{S S} \geq \Pi_{B}^{C C}$ for any value of $q$.

This lemma stipulates that both firms fare better when they produce standard products than when they customize, even if the fixed cost of customization is zero. Obviously, this is due to the reduced competition in outcome SS compared to CC.

Lemma 6 (Outcome SC versus outcome CS). $\Pi_{A}^{S C}+\Pi_{B}^{S C}>\Pi_{A}^{C S}+\Pi_{B}^{C S}$ for any value of $q$.

Intuitively, customization makes the low quality firm more aggressive than it does the high quality firm. Hence, price competition following outcome CS is more fierce compared to that following SC. This is responsible for the result in Lemma 6 that the industry profit is higher when the high quality firm customizes than when the low quality firm customizes.

\subsection{The Endogenous-Timing Game}

Our analysis of the endogenous-timing game applies directly the results in Hamilton and Slutsky (1993). Because the simultaneous game has a unique Nash equilibrium in pure strategies, either the Nash equilibrium is in dominant strategies, or it involves one dominant strategy. ${ }^{6}$ When the Nash equilibrium is in dominant strategies, the order of product type selection in the endogenous-timing game does not matter. This occurs when $K$ is neither in between $c_{1}$ and $c_{2}$ nor in between $r_{1}$ and $r_{2}$. There are multiple subgame perfect equilibria yielding a unique outcome that coincides with the Nash equilibrium of the simultaneous game. More specifically, the following holds.

- If $K \leq \min \left\{c_{1}, c_{2}\right\}$, then both firms select customized products.

- If $K \in\left(\max \left\{c_{1}, c_{2}\right\}, \min \left\{r_{1}, r_{2}\right\}\right]$, then firm A selects a standard product and firm B selects a customized product.

- If $K>\max \left\{r_{1}, r_{2}\right\}$, then both firms select standard products.

When the Nash equilibrium of the simultaneous game involves only one dominant strategy, the endogenous-timing game may lead to different customization choices. ${ }^{7}$ This occurs when $K$ is either in between $c_{1}$ and $c_{2}$ or in between $r_{1}$ and $r_{2}$. Hence, it is left to investigate the four regions highlighted in Figure 4. As in Hamilton and Slutsky (1993), we will focus on equilibria in undominated strategies.

\section{Region I}

Here, we have $K \in\left(c_{1}, c_{2}\right]$. The Nash equilibrium of the simultaneous game is (C,C) and only firm $\mathrm{B}$ has a dominant strategy. Applying backward induction to the endogenous-timing game leads to

\footnotetext{
${ }^{6}$ See Lemma I of Hamilton and Slutsky (1993).

${ }^{7}$ See Theorem III of Hamilton and Slutsky (1993).
} 


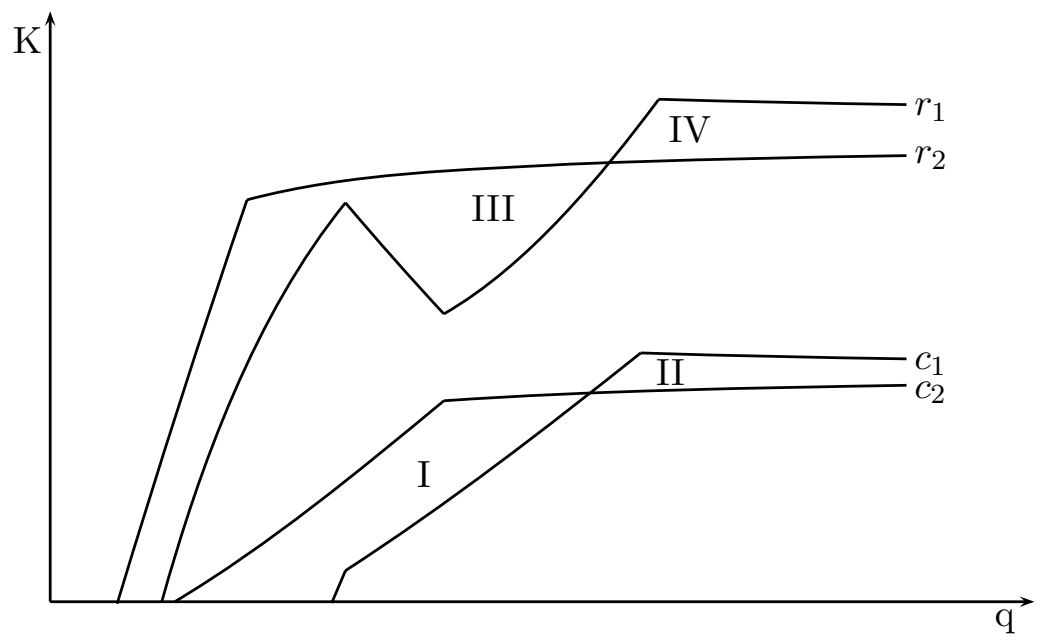

Figure 4: Regions in which NE of the simultaneous game has one dominant strategy

the following $3 \times 3$ payoff matrix for the period 1 strategies:

Firm B

\begin{tabular}{cc|c|c|c} 
& & $\mathrm{S}$ & $\mathrm{C}$ & $\mathrm{W}$ \\
\cline { 2 - 5 } Firm A & $\mathrm{S}$ & $\Pi_{A}^{S S}, \Pi_{B}^{S S}$ & $\Pi_{A}^{S C}, \Pi_{B}^{S C}-K$ & $\Pi_{A}^{S C}, \Pi_{B}^{S C}-K$ \\
\cline { 2 - 5 } & $\mathrm{C}$ & $\Pi_{A}^{C S}-K, \Pi_{B}^{C S}$ & $\Pi_{A}^{C C}-K, \Pi_{B}^{C C}-K$ & $\Pi_{A}^{C C}-K, \Pi_{B}^{C C}-K$ \\
\hline & $\mathrm{W}$ & $\Pi_{A}^{S S}, \Pi_{B}^{S S}$ & $\Pi_{A}^{C C}-K, \Pi_{B}^{C C}-K$ & $\Pi_{A}^{C C}-K, \Pi_{B}^{C C}-K$
\end{tabular}

This matrix deserves a discussion. The upper left $2 \times 2$ submatrix is obvious. Cell (W,W) reflects the fact that $(\mathrm{C}, \mathrm{C})$ is the Nash equilibrium of the simultaneous game. If in period 1 firm A selects its product type and firm B waits, then in period 2 firm B's best response is to customize. This is reflected in the off-diagonal cells of the last column, $(\mathrm{S}, \mathrm{W})$ and $(\mathrm{C}, \mathrm{W})$. If in period 1 firm $\mathrm{B}$ selects its product type and firm A waits, then in period 2 firm A will match firm B's selection (cells (W,S) and $(\mathrm{W}, \mathrm{C})$ in the matrix).

Because $K \in\left(c_{1}, c_{2}\right]$,

$$
\Pi_{A}^{S S}>\Pi_{A}^{C S}-K \text { and } \Pi_{A}^{C C}-K \geq \Pi_{A}^{S C}
$$

W weakly dominates S and C for firm A. From Lemma 5,

$$
\Pi_{B}^{S S}>\Pi_{B}^{C C}-K
$$

holds for any $q$ and $K$ in region I. It follows that firm B will select a standard product against waiting by firm A. The unique Nash equilibrium for matrix (10) in undominated strategies is (W,S). The outcome corresponding to this equilibrium is SS. ${ }^{8}$ Observe that, by Lemma 5 , this outcome is

\footnotetext{
${ }^{8}$ Matrix (10) has two Nash equilibria in weakly dominated strategies (C,C) and (C,W), with outcome CC.
} 
Pareto superior to that in the simultaneous game.

\section{Region II}

In this region, $K \in\left(c_{2}, c_{1}\right]$. The Nash equilibrium of the simultaneous game is (S,C) and only firm $\mathrm{B}$ has a dominant strategy. Applying backward induction leads to the following game matrix for period 1:

\section{Firm B}

\begin{tabular}{cc|c|c|c} 
& & $\mathrm{S}$ & $\mathrm{C}$ & $\mathrm{W}$ \\
\cline { 2 - 5 } Firm A & $\mathrm{S}$ & $\Pi_{A}^{S S}, \Pi_{B}^{S S}$ & $\Pi_{A}^{S C}, \Pi_{B}^{S C}-K$ & $\Pi_{A}^{S C}, \Pi_{B}^{S C}-K$ \\
\cline { 2 - 5 } & $\mathrm{C}$ & $\Pi_{A}^{C S}-K, \Pi_{B}^{C S}$ & $\Pi_{A}^{C C}-K, \Pi_{B}^{C C}-K$ & $\Pi_{A}^{C C}-K, \Pi_{B}^{C C}-K$ \\
\hline & $\mathrm{W}$ & $\Pi_{A}^{C S}-K, \Pi_{B}^{C S}$ & $\Pi_{A}^{S C}, \Pi_{B}^{S C}-K$ & $\Pi_{A}^{S C}, \Pi_{B}^{S C}-K$
\end{tabular}

This matrix differs from matrix (10) in the last row. If in period 1 firm B selects its product type and firm A waits, then in period 2 firm A's best reply will be to make a different selection from firm B's. This is reflected in cells $(\mathrm{W}, \mathrm{S})$ and $(\mathrm{W}, \mathrm{C})$. Cell $(\mathrm{W}, \mathrm{W})$ is due to the fact that $(\mathrm{S}, \mathrm{C})$ is the Nash equilibrium of the simultaneous game.

It follows from $K \in\left(c_{2}, c_{1}\right]$ that waiting is a weakly dominant strategy for firm A. We show in the Appendix that for any $q$ and $K$ in region II

$$
\Pi_{B}^{S C}-K>\Pi_{B}^{C S}
$$

Hence, firm B will choose either $\mathrm{C}$ or $\mathrm{W}$ against firm A's waiting. The Nash equilibria for matrix (11) in undominated strategies are $(\mathrm{W}, \mathrm{C})$ and $(\mathrm{W}, \mathrm{W})$. Both yield outcome SC, the same as in the simultaneous game.

\section{Region III}

In this region, $K \in\left(r_{1}, r_{2}\right]$. The Nash equilibrium of the simultaneous game is (S,S) and only firm $A$ has a dominant strategy. Applying backward induction leads to the following matrix for period 1:

Firm B

\begin{tabular}{cc|c|c|c} 
& & $\mathrm{S}$ & $\mathrm{C}$ & $\mathrm{W}$ \\
\cline { 2 - 5 } Firm A & $\mathrm{S}$ & $\Pi_{A}^{S S}, \Pi_{B}^{S S}$ & $\Pi_{A}^{S C}, \Pi_{B}^{S C}-K$ & $\Pi_{A}^{S S}, \Pi_{B}^{S S}$ \\
\cline { 2 - 5 } & $\mathrm{C}$ & $\Pi_{A}^{C S}-K, \Pi_{B}^{C S}$ & $\Pi_{A}^{C C}-K, \Pi_{B}^{C C}-K$ & $\Pi_{A}^{C C}-K, \Pi_{B}^{C C}-K$ \\
\hline & $\mathrm{W}$ & $\Pi_{A}^{S S}, \Pi_{B}^{S S}$ & $\Pi_{A}^{S C}, \Pi_{B}^{S C}-K$ & $\Pi_{A}^{S S}, \Pi_{B}^{S S}$
\end{tabular}

Here, firm B has a weakly dominant strategy to wait. Because

$$
\Pi_{A}^{S S}>\Pi_{A}^{C C}-K
$$


holds for any $q$ and $K$ in region III by Lemma 5 , firm A will choose either $\mathrm{S}$ or W against waiting by firm B. The Nash equilibria in undominated strategies for matrix (13) are (S,W) and (W,W). Both yield outcome SS, the same as in the simultaneous game.

\section{Region IV}

Region IV turns out to be the most interesting. In this region, $K \in\left(r_{2}, r_{1}\right]$. The Nash equilibrium of the simultaneous game is (S,C) and only firm A has a dominant strategy. Applying backward induction leads to the following matrix for period 1 :

Firm B

\begin{tabular}{cc|c|c|c} 
& & $\mathrm{S}$ & $\mathrm{C}$ & $\mathrm{W}$ \\
\cline { 2 - 5 } Firm A & $\mathrm{S}$ & $\Pi_{A}^{S S}, \Pi_{B}^{S S}$ & $\Pi_{A}^{S C}, \Pi_{B}^{S C}-K$ & $\Pi_{A}^{S C}, \Pi_{B}^{S C}-K$ \\
\cline { 2 - 5 } & $\mathrm{C}$ & $\Pi_{A}^{C S}-K, \Pi_{B}^{C S}$ & $\Pi_{A}^{C C}-K, \Pi_{B}^{C C}-K$ & $\Pi_{A}^{C S}-K, \Pi_{B}^{C S}$ \\
\hline & $\mathrm{W}$ & $\Pi_{A}^{S S}, \Pi_{B}^{S S}$ & $\Pi_{A}^{S C}, \Pi_{B}^{S C}-K$ & $\Pi_{A}^{S C}, \Pi_{B}^{S C}-K$
\end{tabular}

In this matrix, firm $\mathrm{B}$ has a weakly dominant strategy to wait. Firm $\mathrm{A}$ will choose $\mathrm{C}$ against firm B's waiting if

$$
\Pi_{A}^{C S}-K \geq \Pi_{A}^{S C}
$$

or, equivalently,

$$
K \leq \Pi_{A}^{C S}-\Pi_{A}^{S C}=\frac{2}{9} .
$$

In this case $(\mathrm{C}, \mathrm{W})$ is the unique Nash equilibrium in undominated strategies, with outcome CS. Note that firm B is worse off compared to the simultaneous game. ${ }^{9}$ For the other case in which $K>2 / 9,(\mathrm{~S}, \mathrm{~W})$ and $(\mathrm{W}, \mathrm{W})$ are Nash equilibria in undominated strategies. Both yield outcome $\mathrm{SC}$, the same as in the simultaneous game. It is shown in the Appendix that both of the above two subregions of region IV are non-empty. ${ }^{10}$

\section{Summary}

Let us summarize our analysis of the four regions. For the firm that does not have a dominant strategy in the simultaneous game, waiting is a weakly dominant strategy in the endogenous-timing game. Hence, the firm that has a dominant strategy in the simultaneous game (firm B in regions I and II, firm A in regions III and IV) can potentially enhance its position in the endogenous-timing game. Indeed, this firm can secure its equilibrium payoff from the simultaneous game by playing its dominant strategy in period 1 of the endogenous-timing game. However, it can change its payoff by playing its dominated strategy in period 1. It will do so if that is beneficial. Specifically, we have the following.

- The firm that has a dominant strategy in the simultaneous game does not benefit from the

\footnotetext{
${ }^{9}$ This is because $\Pi_{A}^{C S}-K \geq \Pi_{A}^{S C}$ and $\Pi_{A}^{S C}+\Pi_{B}^{S C}>\Pi_{A}^{C S}+\Pi_{B}^{C S}$ (Lemma 6) imply $\Pi_{B}^{C S}-K<\Pi_{B}^{S C}$.

${ }^{10}$ Matrix (14) has two Nash equilibria in weakly dominated strategies (S,C) and (W,C), with outcome SC.
} 


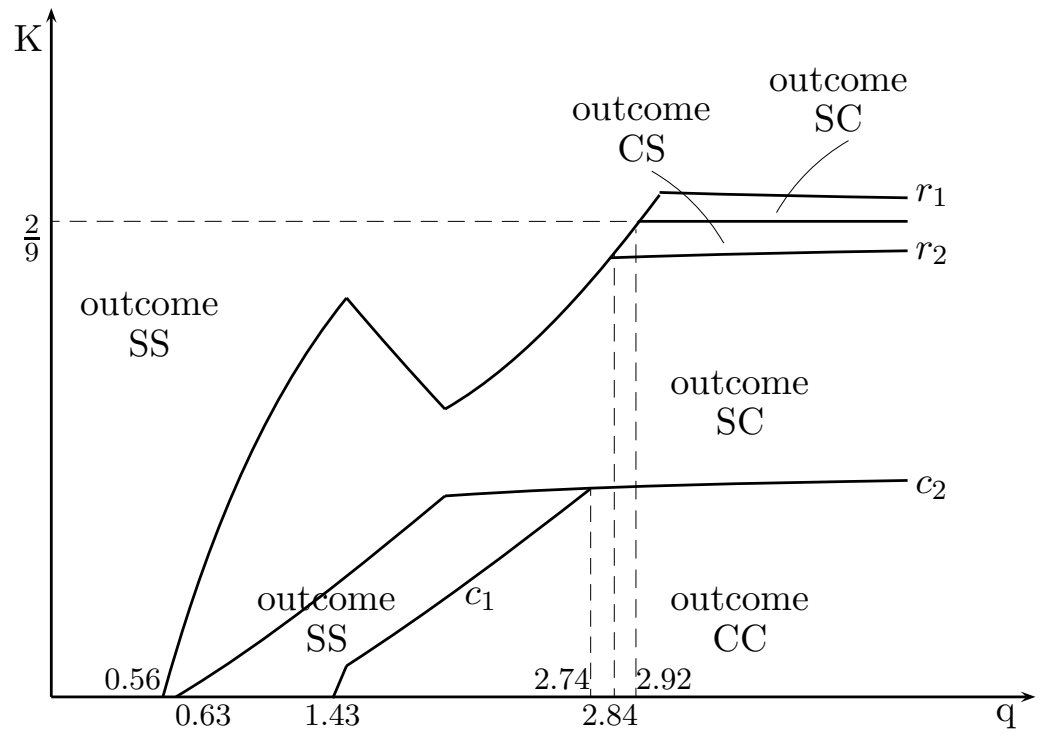

Figure 5: Customization choices in the endogenous-timing game

endogenous-timing game in regions II, III and the upper portion of IV. Both firms receive the same payoffs in the endogenous-timing game as in the simultaneous game.

- The firm that has a dominant strategy in the simultaneous game benefits from the endogenoustiming game in regions I and the lower portion of IV. The other firm is also better off in region I, but is worse off in the lower portion of region IV.

The next proposition points out the differences between equilibrium outcomes in the simultaneous and endogenous-timing games.

Proposition 2 (Endogenous-timing game versus simultaneous game). The following comparison holds between the simultaneous and endogenous-timing games.

(i) If $0.63<q \leq 2.74$ and $K \in\left(\max \left\{0, c_{1}\right\}, c_{2}\right]$, then the endogenous-timing game results in both firms selecting standard products, whereas in the simultaneous game both firms customize.

(ii) If $q>2.84$ and $K \in\left(r_{2}, \min \left\{r_{1}, 2 / 9\right\}\right]$, then the endogenous-timing game results in firm $A$ selecting a customized product and firm B selecting a standard product, whereas in the simultaneous game the opposite occurs.

For all other parameter configurations the two games yield the same outcome.

Combining the results in this proposition with Figure 3 leads to Figure 5, which presents the equilibrium customization choices in the endogenous-timing game.

It follows from Propositions 1 and 2 that both quality difference and endogenous timing play important roles in determining the equilibrium outcome. Indeed, if the quality difference is small $(q \leq 0.56)$ then no firm customizes in both simultaneous and endogenous-timing games, regardless 
of the level of $K$. However, when the quality difference is large, firms are sufficiently differentiated. Customization by one or both firms may occur and the simultaneous and endogenous-timing games do not always lead to the same outcome. In the endogenous-timing game, the firm that does not have a dominant strategy in the simultaneous game has an incentive to wait, and the firm that has a dominant strategy can achieve a different outcome from that of the simultaneous game by playing its dominated strategy in period 1.

\section{Conclusion}

In this paper we have studied customization in a model with two firms that are initially differentiated horizontally and vertically. Customization affects horizontal but not vertical differentiation. When making their customization decisions, the firms play a two-period endogenous-timing game, in which each firm either selects its product type in period 1 or postpones this decision to period 2. After the product types are selected, the firms engage in a price competition.

Both this paper and Loginova and Wang (2008) study customization in the presence of quality differentiation. The two studies together show that quality difference affects the firms' equilibrium customization choices. In particular, no firm will customize if the quality difference is small, regardless of the fixed cost of customization. For sufficiently large quality differences, customization by one or both firms may occur. Because the high quality firm benefits more from customization than the low quality firm, the high quality firm is more likely to customize.

The present study complements Loginova and Wang (2008) by showing that the timing of customization can be used as a strategic tool by the firms. Endogenous timing in customization choices may enable the firms to achieve a Pareto superior outcome by avoiding a price war that would follow customization by both firms. This happens when the high quality firm is the only firm with a dominant strategy in the simultaneous game. While in the simultaneous game the low quality firm never customizes alone, in the endogenous-timing game it can obtain an advantage by becoming the first and only firm to customize. This happens when the low quality firm is the only firm with a dominant strategy in the simultaneous game. 


\section{References}

[1] Alexandrov, Alexei, 2008, "Fat Products," Journal of Economics and Management Strategy, 17(1), pp. 67-95.

[2] Amir, Rabah, and Anna Stepanova, 2006, "Second-Mover Advantage and Price Leadership in Bertrandt Duopoly," Games and Economic Behavior, 55(1), pp.1-20.

[3] Bernhardt, Dan, Qihong Liu, and Konstantinos Serfes, 2007, "Product Customization," European Economic Review, 51(6), pp. 1396-1422.

[4] Dewan, Rajiv, Bing Jing, and Abraham Seidmann, 2003, "Product Customization and Price Competition on the Internet," Management Science, 49(8), pp. 1055-1070.

[5] Economides, Nicholas, 1989, "Quality Variations and Maximal Variety Differentiation," Regional Science and Urban Economics, 19(1), pp. 21-29.

[6] Hamilton, Jonathan H., and Steven M. Slutsky, 1990, "Endogenous Timing in Duopoly Games: Stackelberg or Cournot Equilibria," Games and Economic Behavior, 2(1), pp. 29-46.

[7] Hamilton, Jonathan H., and Steven M. Slutsky, 1993, "Endogenizing the Order of Moves in Matrix Games," Theory and Decision, 34(1), pp. 47-62.

[8] Loginova, Oksana, and Henry X. Wang, 2008, "Mass Customization with Vertically Differentiated Products," University of Missouri, http://web.missouri.edu/ Ioginovao/.

[9] Matsumura, Toshihiro, 1999, "Quantity-Setting Oligopoly with Endogenous Sequencing," International Journal of Industrial Organization, 17(2), 289-296.

[10] Mendelson, Haim, and Ali K. Parlaktürk, 2008, "Competitive Customization," Manufacturing \& Service Operations Management, 10(3), 377-390.

[11] Neven, Damien, and Jacques-François Thisse, 1990, "On Quality and Variety Competition," J.J. Gabszewicz, J.-F. Richard, and L.A. Wolsy (eds.), in Economic Decision-Making: Games, Econometrics, and Optimization, Amsterdam: North-Holland, pp. 175-199.

[12] Salop, Steven C., 1979, "Monopolistic Competition with Outside Goods," Bell Journal of Economics, 10(1), pp.141-156.

[13] Syam, Niladri B., and Nanda Kumar, 2006, "On Customized Goods, Standard Goods, and Competition," Marketing Science, 25(5), pp. 525-537.

[14] Syam, Niladri B., Ranran Ruan, and James D. Hess, 2005, "Customized Products: A Competitive Analysis," Marketing Science, 24(4), pp. 569-584. 


\section{Appendix}

Proofs of Lemmas 1 through 4 appear in Loginova and Wang (2008). (See the Appendix for the referees at the end of this manuscript.)

Proof of (9). The expressions for $c_{1}, c_{2}, r_{1}$, and $r_{2}$ as functions of $q$ follow immediately from Lemmas 1 through 4 ,

$$
\begin{gathered}
c_{1}= \begin{cases}\left(\frac{2}{3}-\frac{1}{6} q\right)^{2}-\frac{1}{2}\left(1-\frac{1}{6} q\right)^{2}, & \text { if } q \leq 1 \\
\frac{1}{q}\left(\frac{1}{3} q+\frac{1}{6}\right)^{2}-\frac{1}{2}\left(1-\frac{1}{6} q\right)^{2}, & \text { if } q \in\left(1, \frac{3}{2}\right] \\
\frac{1}{q}\left(\frac{1}{3} q+\frac{1}{6}\right)^{2}-\frac{1}{q}\left(\frac{1+\sqrt{1+16 q}}{8}\right)^{3}, & \text { if } q \in\left(\frac{3}{2}, 3\right] \\
\frac{1}{q}\left(\frac{1}{3} q+\frac{1}{6}\right)^{2}-\frac{1}{9} q=\frac{1}{9}+\frac{1}{36 q}, & \text { if } q>3\end{cases} \\
c_{2}= \begin{cases}\frac{1}{9} q-\left(\frac{1}{3}-\frac{1}{6} q\right)^{2}, & \text { if } q \leq \frac{1}{2} \\
\frac{1}{9} q-\frac{1}{16} \sqrt{2 q}, & \text { if } q \in\left(\frac{1}{2}, 2\right] \\
\frac{1}{9} q-\frac{1}{q}\left(\frac{1}{3} q-\frac{1}{6}\right)^{2}=\frac{1}{9}-\frac{1}{36 q}, & \text { if } q>2\end{cases} \\
r_{1}= \begin{cases}\left(\frac{2}{3}+\frac{1}{6} q\right)^{2}-\frac{1}{2}\left(1+\frac{1}{6} q\right)^{2}, & \text { if } q \leq \frac{1}{2} \\
\frac{9}{16} \sqrt{2 q}-\frac{1}{2}\left(1+\frac{1}{6} q\right)^{2}, & \text { if } q \in\left(\frac{1}{2}, \frac{3}{2}\right] \\
\frac{9}{16} \sqrt{2 q}-\left(1-\frac{1}{q}\left(\frac{1+\sqrt{1+16 q}}{8}\right)^{2}\right) \frac{-5+3 \sqrt{1+16 q}}{8}, & \text { if } q \in\left(\frac{3}{2}, 2\right] \\
\frac{1}{q}\left(\frac{2}{3} q+\frac{1}{6}\right)^{2}-\left(1-\frac{1}{q}\left(\frac{1+\sqrt{1+16 q}}{8}\right)^{2}\right) \frac{-5+3 \sqrt{1+16 q}}{8}, & \text { if } q \in(2,3] \\
\frac{1}{q}\left(\frac{2}{3} q+\frac{1}{6}\right)^{2}-\frac{4}{9} q=\frac{2}{9}+\frac{1}{36 q}, & \text { if } q>3\end{cases}
\end{gathered}
$$

and

$$
r_{2}= \begin{cases}\frac{4}{9} q-\left(\frac{1}{3}+\frac{1}{6} q\right)^{2}, & \text { if } q \leq 1 \\ \frac{4}{9} q-\frac{1}{q}\left(\frac{2}{3} q-\frac{1}{6}\right)^{2}=\frac{2}{9}-\frac{1}{36 q}, & \text { if } q>1\end{cases}
$$

Tedious but straightforward numerical calculations confirm that $c_{i}<r_{j}$ for $i, j=1,2$ and any given value of $q$.

Proof of Lemma 5. The expressions for $\Pi_{A}^{S S}$ and $\Pi_{B}^{S S}$ as functions of $q$ are given in Lemma 1 . Numerical calculations confirm that $\Pi_{A}^{S S}>\Pi_{A}^{C C}=q / 9$ and $\Pi_{B}^{S S}>\Pi_{B}^{C C}=4 q / 9$ for $q<3$. For $q \geq 3, \Pi_{A}^{S S}=\Pi_{A}^{C C}$ and $\Pi_{B}^{S S}=\Pi_{B}^{C C}$.

Proof of Lemma 6. It follows from Lemmas 2 and 3 that

$$
\Pi_{A}^{C S}+\Pi_{B}^{C S}= \begin{cases}\frac{5}{9}-\frac{1}{9} q+\frac{1}{18} q^{2}, & \text { if } q \leq 1 \\ \frac{1}{q}\left(\frac{5}{9} q^{2}-\frac{1}{9} q+\frac{1}{18}\right), & \text { if } q>1\end{cases}
$$

and

$$
\Pi_{A}^{S C}+\Pi_{B}^{S C}= \begin{cases}\frac{5}{9}+\frac{1}{9} q+\frac{1}{18} q^{2}, & \text { if } q \leq 1 / 2 \\ \frac{5}{8} \sqrt{2 q}, & \text { if } q \in\left(\frac{1}{2}, 2\right] \\ \frac{1}{q}\left(\frac{5}{9} q^{2}+\frac{1}{9} q+\frac{1}{18}\right), & \text { if } q>2\end{cases}
$$


Obviously

$$
\Pi_{A}^{C S}+\Pi_{B}^{C S}>\Pi_{A}^{S C}+\Pi_{B}^{S C}
$$

holds for $q \leq 1 / 2$ and $q>2$. Straightforward calculations show that the inequality also holds for $q \in(1 / 2,2]$.

Proof of (12). It suffices to show that

$$
\Pi_{B}^{S C}-\Pi_{B}^{C S}>c_{1}
$$

holds for $q>2.74$. Lemmas 2 and 3 imply $\Pi_{B}^{S C}-\Pi_{B}^{C S}=4 / 9$. Straightforward calculations confirm that $4 / 9>c_{1}$ for $q>2.74$.

Proof of non-emptiness of the two subregions of region $I V$. For $q>3$,

$$
r_{1}=\frac{2}{9}+\frac{1}{36 q} \quad \text { and } \quad r_{2}=\frac{2}{9}-\frac{1}{36 q}
$$

Hence,

$$
r_{2}<\frac{2}{9}<r_{1}
$$

from which non-emptiness of the two subregions of region IV follows.

Proof of Proposition 2. The results follow immediately from Proposition 1 and the discussion of the four regions preceding this proposition. 


\section{Appendix for the Referees}

Proof of Lemma 1. Each case is proven in turn.

(i) Consider $q \leq 3 / 2$ and suppose the indifference line (5) intersects the unit square as shown in Figure 2(a). Straightforward algebra implies

$$
D_{A}\left(p_{A}, p_{B}\right)=\frac{1}{2}\left(1-\frac{1}{2} q+p_{B}-p_{A}\right) \quad \text { and } \quad D_{B}\left(p_{A}, p_{B}\right)=\frac{1}{2}\left(1+\frac{1}{2} q+p_{A}-p_{B}\right)
$$

in this case. The profit maximizing first-order conditions yield the equilibrium prices and profits as in part (i) of the lemma. It is left to verify that under these prices the indifference line intersects the top and bottom sides of the unit square. Algebraically, $\widehat{x}(1) \geq 0$ and $\widehat{x}(0) \leq 1$. Indeed,

$$
\begin{gathered}
\widehat{x}(1)=\frac{1}{2}\left(1-q+p_{B}^{S S}-p_{A}^{S S}\right)=\frac{1}{2}\left(1-\frac{2}{3} q\right) \geq 0, \\
\widehat{x}(0)=\frac{1}{2}\left(1+p_{B}^{S S}-p_{A}^{S S}\right)=\frac{1}{2}\left(1+\frac{1}{3} q\right)<1
\end{gathered}
$$

hold for $q \leq 3 / 2$. In fact, $\widehat{x}(1)=0$ when $q=3 / 2$.

(ii) Consider $3 / 2<q \leq 3$ and suppose the indifference line (5) intersects the unit square as shown in Figure 2(b). The firms' demand functions are

$$
D_{A}\left(p_{A}, p_{B}\right)=\frac{1}{4 q}\left(1+p_{B}-p_{A}\right)^{2} \quad \text { and } \quad D_{B}\left(p_{A}, p_{B}\right)=1-\frac{1}{4 q}\left(1+p_{B}-p_{A}\right)^{2} .
$$

The first-order conditions yield the equilibrium prices and profits as in part (ii) of the lemma. Under these prices the indifference line intersects the left and bottom sides of the unit square. Algebraically, $\widehat{x}(1) \leq 0$ and $\widehat{x}(0) \in[0,1]$. Indeed,

$$
\begin{aligned}
& \widehat{x}(1)=\frac{1}{2}\left(1-q+\frac{1}{4}(-3+\sqrt{1+16 q})\right)<0, \\
& \widehat{x}(0)=\frac{1}{2}\left(1+\frac{1}{4}(-3+\sqrt{1+16 q})\right) \in(0,1]
\end{aligned}
$$

hold for $3 / 2<q \leq 3$. Note that $\widehat{x}(1)=0$ when $q=3 / 2$ and $\widehat{x}(0)=1$ when $q=3$.

(iii) Consider $q>3$ and suppose the indifference line (5) intersects the unit square as shown in Figure 2(c). The firms' demand functions are

$$
D_{A}\left(p_{A}, p_{B}\right)=\frac{1}{q}\left(p_{B}-p_{A}\right) \quad \text { and } \quad D_{B}\left(p_{A}, p_{B}\right)=\frac{1}{q}\left(q+p_{A}-p_{B}\right)
$$

The first-order conditions yield the equilibrium prices and profits as in part (iii) of the lemma. Under these prices the indifference line intersects the right and left sides of the unit square. 
Algebraically, $\widehat{x}(1) \leq 0$ and $\widehat{x}(0) \geq 1$. Indeed,

$$
\widehat{x}(1)=\frac{1}{2}\left(1-\frac{2}{3} q\right)<0 \quad \text { and } \quad \widehat{x}(0)=\frac{1}{2}\left(1+\frac{1}{3} q\right)>1
$$

hold for $q>3$. Note that $\widehat{x}(0)=1$ when $q=3$.

Proof of Lemma 2. Each case is proven in turn.

(i) Consider $q \leq 1$ and suppose the indifference line (6) intersects the unit square as shown in Figure 2(a). The firms' demand functions are

$$
D_{A}\left(p_{A}, p_{B}\right)=1-\frac{1}{2} q+p_{B}-p_{A} \quad \text { and } \quad D_{B}\left(p_{A}, p_{B}\right)=\frac{1}{2} q+p_{A}-p_{B}
$$

The first-order conditions yield the equilibrium prices and profits as in part (i) of the lemma. Under these prices the indifference line intersects the top and bottom sides of the unit square. Algebraically, $\widehat{x}(1) \geq 0$ and $\widehat{x}(0) \leq 1$. Indeed,

$$
\widehat{x}(1)=1-q+p_{B}^{C S}-p_{A}^{C S}=\frac{2}{3}-\frac{2}{3} q \geq 0 \quad \text { and } \quad \widehat{x}(0)=1+p_{B}^{C S}-p_{A}^{C S}=\frac{2}{3}+\frac{1}{3} q<1
$$

hold for $q \leq 1$. Note that $\widehat{x}(1)=0$ and $\widehat{x}(0)=1$ when $q=1$.

(ii) Consider $q>1$ and suppose the indifference line (6) intersects the unit square as shown in Figure 2(c). The firms' demand functions are

$$
D_{A}\left(p_{A}, p_{B}\right)=\frac{1}{q}\left(\frac{1}{2}+p_{B}-p_{A}\right) \quad \text { and } \quad D_{B}\left(p_{A}, p_{B}\right)=\frac{1}{q}\left(q-\frac{1}{2}+p_{A}-p_{B}\right) .
$$

The first-order conditions yield the equilibrium prices and profits as in part (ii) of the lemma. Under these prices the indifference line intersects the right and left sides of the unit square. Algebraically, $\widehat{x}(1) \leq 0$ and $\widehat{x}(0) \geq 1$. Indeed,

$$
\widehat{x}(1)=\frac{2}{3}-\frac{2}{3} q<0 \quad \text { and } \quad \widehat{x}(0)=\frac{2}{3}+\frac{1}{3} q>1
$$

hold for $q>1$. Note that $\widehat{x}(1)=0$ and $\widehat{x}(0)=1$ when $q=1$.

Proof of Lemma 3. Each case is proven in turn.

(i) Consider $q \leq 1 / 2$ and suppose the indifference line (7) intersects the unit square as shown in Figure 2(a). The firms' demand functions are

$$
D_{A}\left(p_{A}, p_{B}\right)=-\frac{1}{2} q+p_{B}-p_{A} \quad \text { and } \quad D_{B}\left(p_{A}, p_{B}\right)=1+\frac{1}{2} q+p_{A}-p_{B} .
$$


The first-order conditions yield the equilibrium prices and profits as in part (i) of the lemma. Under these prices the indifference line intersects the top and bottom sides of the unit square. Algebraically, $\widehat{x}(1) \geq 0$ and $\widehat{x}(0) \leq 1$. Indeed,

$$
\widehat{x}(1)=-q+p_{B}^{S C}-p_{A}^{S C}=\frac{1}{3}-\frac{2}{3} q \geq 0 \quad \text { and } \quad \widehat{x}(0)=p_{B}^{S C}-p_{A}^{S C}=\frac{1}{3}+\frac{1}{3} q<1
$$

hold for $q \leq 1 / 2$. Note that $\widehat{x}(1)=0$ when $q=1 / 2$.

(ii) Consider $1 / 2<q \leq 2$ and suppose the indifference line (7) intersects the unit square as shown in Figure 2(b). The firms' demand functions are

$$
D_{A}\left(p_{A}, p_{B}\right)=\frac{1}{2 q}\left(p_{B}-p_{A}\right)^{2} \quad \text { and } \quad D_{B}\left(p_{A}, p_{B}\right)=1-\frac{1}{2 q}\left(p_{B}-p_{A}\right)^{2} .
$$

The first-order conditions yield the equilibrium prices and profits as in part (ii) of the lemma. Under these prices the indifference line intersects the left and bottom sides of the unit square. Algebraically, $\widehat{x}(1) \leq 0$ and $\widehat{x}(0) \in[0,1]$. Indeed,

$$
\widehat{x}(1)=-q+\frac{1}{2} \sqrt{2 q}<0 \text { and } \widehat{x}(0)=\frac{1}{2} \sqrt{2 q} \in(0,1]
$$

hold for $1 / 2<q \leq 2$. Note that $\widehat{x}(1)=0$ when $q=1 / 2$ and $\widehat{x}(0)=1$ when $q=2$.

(iii) Consider $q>2$ and suppose the indifference line (7) intersects the unit square as shown in Figure 2(c). The firms' demand functions are

$$
D_{A}\left(p_{A}, p_{B}\right)=\frac{1}{q}\left(-\frac{1}{2}+p_{B}-p_{A}\right) \quad \text { and } \quad D_{B}\left(p_{A}, p_{B}\right)=\frac{1}{q}\left(q+\frac{1}{2}+p_{A}-p_{B}\right) .
$$

The first-order conditions yield the equilibrium prices and profits as in part (iii) of the lemma. Under these prices the indifference line intersects the right and left sides of the unit square. Algebraically, $\widehat{x}(1) \leq 0$ and $\widehat{x}(0) \geq 1$. Indeed,

$$
\widehat{x}(1)=\frac{1}{3}-\frac{2}{3} q<0 \quad \text { and } \quad \widehat{x}(0)=\frac{1}{3}+\frac{1}{3} q>1
$$

hold for $q>2$. Note that $\widehat{x}(0)=1$ when $q=2$. 